Elsevier required licence: (C) <2017>. This manuscript version is made available under the CC-BY-NC-ND 4.0 license http://creativecommons.org/licenses/by-nc-nd/4.0/ 


\title{
Prediction Market Bias under Risk Aversion and Heterogeneous Beliefs
}

\author{
Xue-Zhong He* Nicolas Treich ${ }^{\dagger}$
}

\begin{abstract}
This paper characterizes prediction market bias when risk averse traders have heterogeneous beliefs in probabilities. For a binary prediction market, we show that the equilibrium state prices equal the mean beliefs of the states if and only if the utility function is logarithmic. More importantly, we provide a necessary and sufficient condition for prediction market bias that the state prices are systematically below or above the mean beliefs of the state. Consequently we provide a rational explanation to the favorite-longshot bias. The results demonstrate how prediction market bias depends critically on the risk preferences and heterogeneous beliefs of traders.
\end{abstract}

${ }^{*}$ Corresponding author. University of Technology Sydney, Business School, Finance Discipline Group, Australia. Email: tony.he1@uts.edu.au

${ }^{\dagger}$ Toulouse School of Economics (LERNA-INRA and IDEI), Toulouse, France. Corresponding author. Email: ntreich@toulouse.inra.fr 


\title{
Prediction Market Bias under Risk Aversion and Heterogeneous Beliefs
}

\begin{abstract}
This paper characterizes prediction market bias when risk averse traders have heterogeneous beliefs in probabilities. For a binary prediction market, we show that the equilibrium state prices equal the mean beliefs of the states if and only if the utility function is logarithmic. More importantly, we provide a necessary and sufficient condition for prediction market bias that the state prices are systematically below or above the mean beliefs of the state. Consequently we provide a rational explanation to the favorite-longshot bias. The results demonstrate how prediction market bias depends critically on the risk preferences and heterogeneous beliefs of traders.
\end{abstract}

Keywords: Prediction market, heterogeneous beliefs, risk aversion, favorite-longshot bias.

JEL: D81, D84, G11, G12 


\section{Introduction}

The measurement of probabilistic beliefs of traders has become increasingly common both in academia and in practice of prediction (Manski 2004, Wolfers and Zitzewitz 2004, Surowiecki 2005, Armantier and Treich 2013). In particular, prediction markets are now considered as one of the most efficient tool to elicit people's beliefs. ${ }^{1}$ They have been repeatedly used to predict the outcome of political elections, like with the Iowa electronic market (www.biz.uiowa.edu/iem/). They are also increasingly used by private companies like Microsoft, Google and Chevron for instance to elicit their employees' beliefs about future sales or industry trends.

Technically, prediction markets are simple financial markets in which traders bet on the outcomes of uncertain events. Asset prices in prediction markets are typically interpreted as probabilities. For instance, Arrow et al. (2008) introduce prediction markets as follows: "Consider a contract that pays $\$ 1$ if Candidate $X$ wins the presidential election in 2008. If the market price of an $X$ contract is currently 53 cents, an interpretation is that the market 'believes' $X$ has a 53\% chance of winning" (Arrow et al. 2008).

Given the development of prediction markets, it is important to better understand when prediction markets are expected to be biased at predicting future events. We say that the prediction market is biased when its market equilibrium state price is different from the mean probabilities of traders' beliefs of the state (and unbiased otherwise). ${ }^{2}$ In this paper, we consider a simple prediction market in which risk averse traders have heterogeneous beliefs in probabilities of different states and examine under which condition the prediction market is biased. In other words, we study when the prediction market is

\footnotetext{
${ }^{1}$ See for instance Hahn and Tetlock (2006). Prediction markets have been used to forecast market capitalization prior to an IPO (Berg et al. 2009), to test double auction in complex environments with few traders (Healy et al. 2010), and to evaluate the information aggregation and manipulation behavior (Jian and Sami 2012). See Goel, Reeves and Pennock (2009) for a more contrasted view about the performance of prediction markets.

${ }^{2}$ This definition is consistent with the early economic papers on prediction markets (Slovic 2000 Forsythe et al. 1992, Hanson 2006 and Arrow et al. 2008) that compared the equilibrium price to the mean beliefs of traders.
} 
not well calibrated on average (Page and Clemen 2013).

This paper makes two main contributions to the literature. Firstly, we derive the exact necessary and sufficient condition for prediction market to be unbiased for general distributions of beliefs. Specifically, we show that the binary prediction market is unbiased for all distributions of beliefs on the states if and only if the utility function is logarithmic. We also provide a sufficient condition on the prediction market to be unbiased for all utility functions. In addition we present several examples in which the (joint) distributions of traders' beliefs, wealth and risk preferences lead to a systematic violation of the unbiased prediction market. Secondly, when prediction market is biased, we provide indications about the direction of the bias. Most significantly, we exhibit the necessary and sufficient condition for the equilibrium price of a state to be always below/above the mean belief of the state for all symmetric belief distributions, depending on risk preferences and the mean beliefs. More precisely, for all symmetric beliefs, there exists a favorite-longshot bias, meaning that the high-likelihood events (or states with the mean beliefs of the states above 1/2) are underpriced and the low-likelihood events (or states with the mean beliefs of the states below $1 / 2$ ) are overpriced, if and only if the traders' risk preferences are such that twice absolute risk aversion is less than absolute prudence. Under constant relative risk aversion (CRRA) with risk aversion coefficient $\gamma$ this condition is equivalent to $\gamma<1$. That is, when traders are less risk averse (than logarithmic utility), we provide a rationale to the well documented favorite-longshot bias (Ali 1977). In contrast, there is a reverse favorite-longshot bias when the utility functions of traders are either CARA or CRRA with $\gamma>1$. In a discussion on the extension of two state to many states prediction market, we show with the help of an example that the equilibrium state price not only depends on the distribution of beliefs about that state, but also about other states.

There is already a significant literature on the favorite-longshot bias in prediction markets. Started with Ali (1977), many models have been developed to explain the favorite-longshot bias, including Gjerstad (2004), Manski (2006), Wolfers and Zitzewitz (2006), and Page and Clemen (2013). When traders are risk neutral and have limited investment budgets, Manski (2006) presents a first formal analysis of this question. He 
shows that the equilibrium price of the prediction market can differ from the mean beliefs of traders. When traders are risk averse, Wolfers and Zitzewitz (2006) show theoretically that the prediction market is unbiased when the utility function of traders is logarithmic. Moreover, Wolfers and Zitzewitz (2006) explore numerically how the equilibrium price is affected by belief heterogeneity for several utility functions and several beliefs distributions. ${ }^{3}$ In this paper, we follow Manski (2006), Gjerstad (2004) and Wolfers and Zitzewitz (2006) and show that the logarithmic utility is not only sufficient but also necessary to ensure the prediction market to be unbiased for all belief distributions. More interestingly, we provide the necessary and sufficient condition theoretically for the equilibrium prices to be systemically biased to the mean beliefs of traders for all symmetric belief distributions. This condition depends critically on the risk preferences and the mean beliefs. It provides a theoretical foundation to the numerical results in Wolfers and Zitzewitz (2006) and a complete characteristization of the theoretical results in Gjerstad (2004).

This paper is also related to but different from some recent papers providing an informational explanation to the favorite-longshot bias (Ottaviani and Sorensen, 2009, 2010, 2015). In a game-theoretic model of parimutuel betting, Ottaviani and Sorensen (2009) assume that traders have a common prior but incorporate in a Bayesian fashion the information revealed by the bets placed at the equilibrium. This model is generalized in Ottaviani and Sorensen (2010) to allow for noise and private information. Recently, Ottaviani and Sorensen (2015) study the price underreaction when traders with heterogeneous prior beliefs react to public information. In all these models, Ottaviani and Sorensen derive theoretical conditions leading to the favorite-longshot bias in a binary prediction market. A prediction market is a simple financial market. Therefore this paper is also closely related to the models of asset pricing under heterogeneous beliefs (see, e.g., Varian 1985, Abel 1989, Jouini and Napp 2006 and 2007, Gollier 2007 and Roche 2011). ${ }^{4}$ These

\footnotetext{
${ }^{3}$ See Gjerstad (2004) for theoretical results under constant relative risk aversion (CRRA) utility functions, and some numerical results. See also Fountain and Harrison (2011) for further numerical results with wealth and beliefs heterogeneity. Thaler and Ziemba (1988) extensively discuss the favorite-longshot bias.

${ }^{4}$ For a justification and implications of these models, see for instance the literature survey papers by
} 
models with heterogeneous beliefs have been used to explain various anomalies in financial markets.

We organize the paper as follows. In the next section we introduce a simple binary prediction market model and derive a sufficient condition for the equilibrium state price to be unique. In Section 3, we derive a necessary and sufficient condition for prediction market to be unbiased on the utility function for all heterogeneous beliefs. We also provide a sufficient condition on prediction market to be unbiased for all utility functions. Then in Section 4 we examine the conditions leading to the favorite-longshot bias. Finally in Section 5 we provide a discussion on the generalization of the previous results in binary prediction market to the market with more than two states. The last section concludes. All the proofs are given in the appendices.

\section{The model}

We consider a simple binary prediction market in which risk averse traders can buy and sell a risky asset paying $\$ 1$ if a specific event or state occurs, and nothing otherwise. The main assumption of the model is that the beliefs of the traders about the occurrence of the specific event are heterogeneous. That is, we consider a model in which traders "agree to disagree", and therefore have different prior beliefs across states. Namely, the heterogeneity in beliefs does not come from asymmetric information but rather from intrinsic differences in how traders interpret information. This assumption has been common in economic models of prediction markets cited in the previous section. Moreover, to focus on the specific effect of beliefs' heterogeneity, we assume in all the Propositions of this paper that the utility function and the wealth of traders are homogenous. ${ }^{5}$ In this section, we derive some properties of the individual asset demand, and then of the equilibrium price in this specific model.

Varian (1989), Scheinkman and Xiong (2004) and Hong and Stein (2007).

${ }^{5}$ The effect of heterogeneity in wealth and utility function on prediction market bias is more complicated, see Remark 1 and Example 2 in the next section for a related discussion. 
In our model, each trader maximizes his expected utility based on his own beliefs. Let $u(\cdot)$ be the trader's vNM utility function, which is strictly increasing, strictly concave and three times differentiable. Formally, when a trader decides how much to invest in the financial asset paying $\$ 1$ if the event occurs, he maximizes the following expected utility

$$
\max _{\alpha}[p u(w+\alpha(1-\pi))+(1-p) u(w-\alpha \pi)]
$$

in which $w$ is his initial wealth, $p \in(0,1)$ his subjective probability or belief that the event occurs, $\alpha$ his asset demand and $\pi$ the price of this asset. ${ }^{6}$ The first order condition of this optimization is given by

$$
p(1-\pi) u^{\prime}(w+\alpha(p, \pi)(1-\pi))-(1-p) \pi u^{\prime}(w-\alpha(p, \pi) \pi)=0,
$$

in which $\alpha(p, \pi)$ is the solution of (2). Differentiating equation (2) with respect to $p$,

$$
\begin{gathered}
0=(1-\pi) u^{\prime}(w+\alpha(p, \pi)(1-\pi))+\pi u^{\prime}(w-\alpha(p, \pi) \pi) \\
+\alpha_{p}(p, \pi)\left[p(1-\pi)^{2} u^{\prime \prime}(w+\alpha(p, \pi)(1-\pi))\right. \\
\left.+(1-p) \pi^{2} u^{\prime \prime}(w-\alpha(p, \pi) \pi)\right]
\end{gathered}
$$

we obtain

$$
\alpha_{p}(p, \pi)=\frac{(1-\pi) u^{\prime}(w+\alpha(p, \pi)(1-\pi))+\pi u^{\prime}(w-\alpha(p, \pi) \pi)}{-p(1-\pi)^{2} u^{\prime \prime}(w+\alpha(p, \pi)(1-\pi))-(1-p) \pi^{2} u^{\prime \prime}(w-\alpha(p, \pi) \pi)}>0,
$$

that is, the asset demand increases with belief $p$. Since $\alpha(p, p)=0$, we conclude that $\alpha(p, \pi) \geq 0$ if and only if $p \geq \pi$. Namely, the trader buys (respectively sells) the asset yielding $\$ 1$ when the event occurs and 0 otherwise if and only if he assigns a probability for this event higher (respectively lower) than the asset price.

Let $\widetilde{p}$ be the random variable representing the distribution of beliefs on the event in the population of traders, and let $\pi^{*}$ be the equilibrium price. The equilibrium condition

\footnotetext{
${ }^{6}$ The individual asset demand $\alpha$ can be seen as the net asset demand of one asset in a model with two Arrow-Debreu assets. To see that, let $\alpha_{s}$ and $\pi_{s}$ denote respectively the demand for and the price of Arrow-Debreu assets in state $s=1,2$. The objective can then be written: $\max _{\alpha_{1}, \alpha_{2}}\left[p u\left(w+\alpha_{1}-\pi_{1} \alpha_{1}-\right.\right.$ $\left.\left.\pi_{2} \alpha_{2}\right)+(1-p) u\left(w+\alpha_{2}-\pi_{1} \alpha_{1}-\pi_{2} \alpha_{2}\right)\right]$. Denoting $\alpha=\alpha_{1}-\alpha_{2}$ and observing that $\pi_{1}+\pi_{2}=1$ by arbitrage then leads (with $\pi=\pi_{1}$ ) to (1).
} 
is

$$
E \alpha\left(\widetilde{p}, \pi^{*}\right)=0
$$

in which $E$ denotes the expectation operator with respect to $\widetilde{p}$, the belief distribution of traders on the event. ${ }^{7}$ Our main objective is to compare $\pi^{*}$ to $E \widetilde{p}$. In particular, in Section 3 we derive condition so that prediction market is unbiased when $\pi^{*}=E \widetilde{p}$. Since $\alpha(p, p)=0$, notice immediately that, when $\widetilde{p}$ is degenerate and equal to homogeneous belief $p$ with probability 1 , then $\pi^{*}=p$ and there is no trade at the equilibrium. This is a trivial case always leading to unbiased prediction market. We rule out this case (until Section 5 with more than two states), and consider nondegenerate $\widetilde{p}$ in the following.

It is easy to see that an equilibrium always exists in such a prediction market. Indeed, when $\pi$ tends to 0 (respectively tends to 1$) \alpha(p, \pi)$ becomes positive (respectively negative) for all $p$, so its expectation over $\widetilde{p}$ also becomes positive (respectively negative). Therefore when $\pi$ increases, the function $E \alpha(\widetilde{p}, \pi)$ must go from a positive to a negative region and thus cross zero somewhere in between at least once.

We first discuss the uniqueness of the equilibrium, that is whether $E \alpha(\widetilde{p}, \pi)$ only crosses the origin once. We know that $\alpha(p, \pi)$ has this single crossing property at $\pi=p$. But that does not guarantee that $E \alpha(\widetilde{p}, \pi)$ also has the single crossing property, as illustrated by the following example.

Example 1 (Multiple equilibria): Consider traders with a quadratic utility function $u(w)=-(1-w)^{2}$ for $0 \leq w \leq 1$ and initial wealth $w=1 / 2$. Following the first order condition the optimal asset demand is then equal to $\alpha(p, \pi)=\frac{p-\pi}{2\left(p-2 p \pi+\pi^{2}\right)}$. In a prediction market with only two traders with respective beliefs of the event denoted $p_{1}=0.1$ and $p_{2}=0.9$, the equilibrium condition is equivalent to $9-68 \pi+150 \pi^{2}-100 \pi^{3}=0$. Solving for this equation, it is found that there are three equilibrium prices in this prediction market: $\pi^{*}=(0.235,0.5,0.764)$.

\footnotetext{
${ }^{7}$ The distribution of traders' beliefs can be discrete or continuous.
} 
A sufficient condition for the uniqueness of the equilibrium however is $\alpha_{\pi}(p, \pi)<0$ everywhere. Indeed, this implies that the function $E \alpha(\widetilde{p}, \pi)$ is strictly decreasing in $\pi$, and therefore crosses zero at most once. Differentiating (2) with respect to $\pi$, we have

$$
\begin{aligned}
& \alpha_{\pi}(p, \pi)= \\
& \quad \frac{-p u^{\prime}(w+\alpha(p, \pi)(1-\pi))-(1-p) u^{\prime}(w-\alpha(p, \pi) \pi)}{-p(1-\pi)^{2} u^{\prime \prime}(w+\alpha(p, \pi)(1-\pi))-(1-p) \pi^{2} u^{\prime \prime}(w-\alpha(p, \pi) \pi)} \\
& -\alpha(p, \pi) \frac{p(1-\pi) u^{\prime \prime}(w+\alpha(p, \pi)(1-\pi))-(1-p) \pi u^{\prime \prime}(w-\alpha(p, \pi) \pi)}{-p(1-\pi)^{2} u^{\prime \prime}(w+\alpha(p, \pi)(1-\pi))-(1-p) \pi^{2} u^{\prime \prime}(w-\alpha(p, \pi) \pi)} .
\end{aligned}
$$

The first term is strictly negative but the second term is of ambiguous sign under risk aversion, so that the demand may increase when the price $\pi$ increases, as it is the case in Example 1. We now provide a sufficient condition for uniqueness by ensuring that the second term is also negative. We show that this is the case under decreasing absolute risk aversion (DARA).

Proposition 1 The equilibrium price $\pi^{*}$ is unique if u satisfies the assumption of DARA.

This result is consistent with Proposition 4 of Ottaviani and Sorensen (2015), although it is obtained in a different setting here. The intuition for this result is the following. When the price of an asset increases, there are two effects captured by the two terms of the right hand side of equation (6). First, there is a substitution effect that leads to a decrease in its demand, but there is also a wealth effect that may potentially increase its demand. Intuitively, as the terminal wealth distribution deteriorates, the investor's attitude towards risk may change, and this wealth effect might prove sufficiently strong to increase the demand for the risky asset, as initially shown by Fishburn and Porter (1976) in the case of a first-order stochastic dominance (FSD) shift. Under DARA however, the negative wealth effect leads the trader to be more risk averse, and therefore further decreases the demand for the risky asset. Under constant absolute risk aversion (CARA), there is no wealth effect, and only the first negative effect is at play. Finally, we note that Example 1 features multiple equilibria because the quadratic utility function has increasing absolute risk aversion. 
When there is a unique equilibrium, one can make a simple comment on the effect of a change in the distribution of beliefs on the equilibrium price. Indeed, from the equilibrium condition $E \alpha\left(\widetilde{p}, \pi^{*}\right)=0$ and $\alpha_{p}(p, \pi)>0$, any FSD improvement in the distribution of beliefs must increase the equilibrium price.

\section{A necessary and sufficient condition for unbiased prediction market}

In this section, we characterize the unbiased prediction market by answering the following question: which utility functions lead to unbiased prediction market for any belief distribution? Correspondingly, we provide a necessary and sufficient condition for prediction market to be unbiased.

To answer the question, we consider a logarithmic utility function ${ }^{8} u(w)=\log w$ (which displays DARA). We can obtain a closed-form solution of the first order condition (2):

$$
\alpha(p, \pi)=w \frac{(p-\pi)}{\pi(1-\pi)} .
$$

This implies that the equilibrium condition (5) can simply be written $\pi^{*}=E \widetilde{p}$. This shows that the logarithmic utility function is sufficient for prediction market to be unbiased, a result obtained in Gjerstad (2004) and Wolfers and Zitzewitz (2006). A natural question is whether the utility function must be logarithmic to guarantee prediction market to be unbiased independently of belief distribution among traders or whether this is possible for other utility functions. That is whether $u(w)=\log w$ is also a necessary condition. We show that this is indeed the case.

Proposition 2 We have $\pi^{*}=E \widetilde{p}$ for all belief distributions $\widetilde{p}$ if and only if $u(w)=\log w$.

\footnotetext{
${ }^{8}$ Since the expected utility theory is defined up to an affine transformation, the statement $u(w)=\log w$ should read in fact $u(w)=a \log w+b$ for constants $a(>0)$ and $b$. A similar observation applies for all utility functions used in the Examples and for the results of Section 5.
} 
This result characterizes unbiased prediction market that is independent of belief distribution among traders. It is a knife edge case for the prediction bias examined in the next section. We complement this result with three remarks about its limitation in more general settings.

Remark 1 (Wealth heterogeneity): The result of Proposition 2 cannot be generalized to non-identical wealth, as possible correlation between wealth and beliefs would invalidate the result. Indeed, let $\widetilde{w}$ be the random variable representing wealth heterogeneity. Assuming a logarithmic utility function, we can obtain

$$
\pi^{*}=E \widetilde{p}+\frac{1}{E_{\widetilde{w}} \widetilde{w}} \operatorname{Cov}(\widetilde{p}, \widetilde{w}) .
$$

Therefore there is no utility function that can always ensure prediction market to be unbiased when beliefs and wealth are potentially correlated. Observe that, despite this impossibility result, the direction of the bias can be inferred if the analyst knows the sign of the correlation between beliefs and wealth. The intuition for (7) is that richer individuals invest more, and therefore have more influence on the equilibrium price. Thus, if wealth is positively (respectively negatively) correlated with beliefs, the equilibrium price will be higher (respectively lower). This result is closely related but different from the example with logarithmic preferences of Ottaviani and Sorensen (2015) who show that, even with logarithmic utility, the equilibrium price can underreact to information, leading to biased price from the mean beliefs when traders with heterogeneous prior beliefs react to the information in the same way.

Remark 2 (Stakes): Suppose each trader has a (positive or negative) stake $\Delta$ in the event he predicts, so that he now maximizes over $\alpha$ the following expected utility

$$
p u(w+\Delta+\alpha(1-\pi))+(1-p) u(w-\alpha \pi) .
$$

Then it is easy to understand that the result of Proposition 2 is not guaranteed either. 
Indeed for the logarithmic utility function we have

$$
\alpha(p, \pi)=w \frac{(p-\pi)}{\pi(1-\pi)}-\Delta \frac{\pi(1-p)}{\pi(1-\pi)}
$$

leading to the equilibrium condition

$$
\pi^{*}=\frac{w E \widetilde{p}}{w+\Delta(1-E \widetilde{p})}
$$

The intuition is that when there is a positive (respectively negative) stake, the marginal utility decreases (respectively increases) if the event occurs. As a result, the traders want to transfer wealth to the state in which the event does not occur (respectively occurs), and they typically use the prediction market as a hedging scheme to do this. The consequence is that the equilibrium is biased downward (respectively upward). Observe that if the stakes are individual-dependent but uncorrelated with beliefs, and if their mean across individuals is equal to zero, then we retrieve prediction market to be unbiased under a logarithmic utility function.

The previous proposition provides the condition on the utility function so that market prediction is unbiased for all belief distribution $\widetilde{p}$. We now study the dual problem: what are the conditions on belief distribution $\widetilde{p}$ to ensure prediction market to be unbiased for all utility function $u$ ? We show that this depends on whether the probability distribution of beliefs is symmetric about one half.

Proposition 3 If $\tilde{p}$ is symmetric about $1 / 2$, then $\pi^{*}=E \widetilde{p}$ for all $u$ that imply a unique equilibrium.

The intuition for Proposition 3 is simple. When $\widetilde{p}$ is symmetric about one half, the two states are formally indistinguishable. Therefore it cannot be that the price of an asset yielding one dollar in one state is different from that of an asset yielding one dollar in the other state, implying $\pi^{*}=1 / 2$.

We note, however, that if heterogeneity in individual utility functions is introduced, prediction market bias may arise even when $\widetilde{p}$ is symmetric about $1 / 2$. The intuition is 
essentially the same as the one presented in Remark 1. This is illustrated by the following example which considers heterogeneity over (constant absolute) risk aversion.

Example 2 (Heterogeneous CARA): Let $u_{i}(w)=-e^{-r_{i} w}$ in which $r_{i}>0$ represents the CARA coefficient of trader $i=1,2$ with respective beliefs $p_{1}=0.1$ and $p_{2}=0.9$ of the event. Under positive correlation between beliefs and risk aversion $\left(r_{1}, r_{2}\right)=(1,3)$, we have $\pi^{*}=1 / 4<1 / 2=E \widetilde{p}$, while under negative correlation $\left(r_{1}, r_{2}\right)=(3,1)$, we have $\pi^{*}=3 / 4>1 / 2=E \widetilde{p}$.

We have characterized conditions for prediction market to be unbiased for all belief distribution $\widetilde{p}$ in Proposition 2 and provided a sufficient condition for all utility function $u$ in Proposition 3. We can see that these conditions are rather stringent. We note, however, that one can relax these conditions in the sense that it is possible to find well-chosen pairs $(u, \widetilde{p})$ also yielding unbiased prediction market. This is shown in the following example which uses a specific constant relative risk aversion (CRRA) utility function and a specific nonsymmetric distribution of beliefs.

Example 3 (Unbiased prediction market under CRRA and nonsymmetric beliefs). Consider traders with utility function $u(w)=-1 / w$. Two groups of traders participate in the prediction market: one group has beliefs $p_{1}=p$, and the other group has beliefs $p_{2}=1-p$. Denoting $a$ the proportion of traders in the first group, we have $E \widetilde{p}=a p+(1-a)(1-p)$. Note that the belief distribution among traders may not be symmetric about $1 / 2$. One may then easily obtain that $E \alpha(\tilde{p}, \pi)=0$ implies $\sqrt{\pi(1-\pi)}\{a p+(1-a)(1-p)-\pi\}=0$ leading to $\pi^{*}=E \widetilde{p}$. This example shows a different result from the price underreaction to information obtained in Ottaviani and Sorensen (2015). This illustrates the importance of information and heterogeneous concordant beliefs.

Examples 2 and 3 indicate that symmetric beliefs about $1 / 2$ is neither necessary nor sufficient for prediction market to be unbiased in general, in particular when traders have heterogeneous risk preference. 


\section{A necessary and sufficient condition for the favorite- longshot bias}

In the previous analysis, we have examined under which conditions the prediction market is unbiased in the sense that the equilibrium price $\pi^{*}$ is equal to the mean belief $E \widetilde{p}$. We have seen that these conditions are rather stringent, implying a bias of the equilibrium price to the mean belief is expected in prediction markets in general (Ottaviani and Sorensen, 2015). It is therefore interesting to see how to characterize such bias. In this section, we derive a necessary and sufficient condition for prediction bias, meaning that the equilibrium price $\pi^{*}$ is systematically above or below the mean belief $E \widetilde{p}$.

The analysis developed in this section may provide a rationale for the favorite-longshot bias, namely for the empirical observation that longshots tend to be over-valued and that favorites tend to be under-valued (Ali 1977). More explicitly, consider a horse race with only two horses, and call the first horse the favorite (resp. longshot) if the mean beliefs that this horse wins are such that $E \widetilde{p} \geq 1 / 2$ (resp. $E \widetilde{p} \leq 1 / 2$ ). As we will see, the necessary and sufficient condition so that this horse is under-valued, i.e. $\pi^{*} \leq E \widetilde{p}$, critically depends on whether it is a favorite or a longshot and on the risk aversion of traders. This result is presented in the following Proposition 4 in which $A(w)=-u^{\prime \prime}(w) / u^{\prime}(w)$ denotes the Arrow-Pratt's coefficient of absolute risk aversion and $P(w)=-u^{\prime \prime \prime}(w) / u^{\prime \prime}(w)$ denotes the coefficient of absolute prudence (Kimball 1990). Essentially, these coefficients respectively capture the intensity with which the utility and the decisions are affected by a change in risk.

Proposition 4 We have $\pi^{*} \geq E \widetilde{p}$ for all symmetric beliefs ${ }^{9} \widetilde{p}$ about the mean belief $E \widetilde{p}$ if and only if $(1 / 2-E \widetilde{p})(P(w)-2 A(w)) \geq 0$ for all $w$ and $u$ that imply a unique equilibrium.

\footnotetext{
${ }^{9}$ Here the symmetry is about the mean probability of state 1 ; for example, $\widetilde{p}=(1 / 3-1 / 6,1 / 3,1 / 3+$ $1 / 6$ ) of three traders' beliefs on the probability of state 1 is a symmetric belief about the mean probability $1 / 3$ of state 1 .
} 
The difference between the mean belief $E \widetilde{p}$ and the equilibrium price $\pi^{*}$ therefore depends on whether the mean belief is less than $1 / 2$, and on whether the absolute prudence is greater than twice the absolute risk aversion. The sign of $P-2 A$ is a familiar condition on utility functions derived from comparative statics analysis within expected utility models (Gollier 2001). In particular, for CARA utility functions, $P=A(<2 A)$. For CRRA utility functions $u(x)=x^{1-\gamma} /(1-\gamma), P<2 A$ is equivalent to the constant relative risk aversion $\gamma>1$, while $P>2 A$ is equivalent to $\gamma<1$, and $P=2 A$ is equivalent to $\gamma=1$, which corresponds to the logarithmic utility function.

Figure 1: The equilibrium price $\pi^{*}$ as a function of mean beliefs $\bar{p}$. Under symmetric beliefs, there is a "favorite-longshot bias" for the class of utility functions $u$ satisfying $P(w)>2 A(w)$.

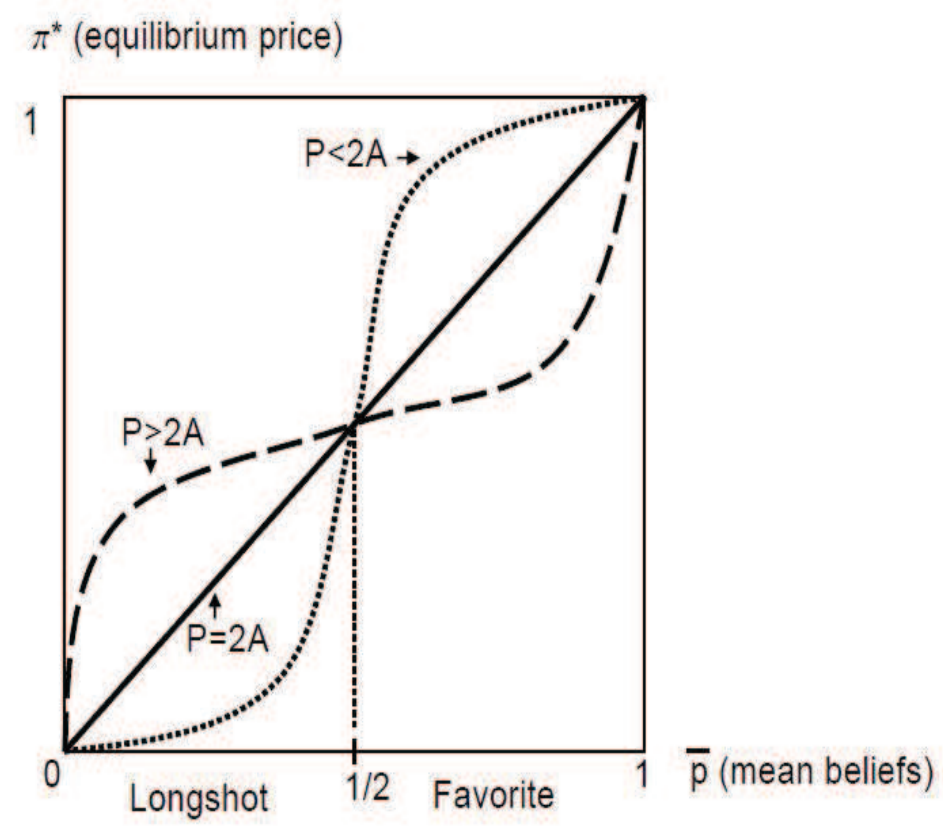

The result in Proposition 4 is illustrated in Figure 1. The horizontal axis represents the mean belief $E \widetilde{p}$ and the vertical axis represents the equilibrium price $\bar{p}=\pi^{*}$. The diagonal therefore represents prediction market accuracy, which holds everywhere if and only if $P=2 A$ (i.e., $u$ is logarithmic). The result therefore shows that there is a favoritelongshot bias if and only if the utility function displays $P>2 A$, or equivalently $\gamma<1$ 
for the CRRA utility functions. This implies that, for all symmetric belief distributions, there is a longshot bias if and only if the traders are less risk averse (than the logarithmic utility function). When the traders are more risk averse, a reverse longshot bias occurs. Therefore, we provide a theoretical foundation for the favorite-longshot bias. The result also generalizes the numerical simulations presented in Wolfers and Zitzewitz (2006) for CRRA utility functions and a set of specific distributions of beliefs.

Observe that the result in Proposition 4 is consistent with Propositions 2 and 3. Indeed, this result shows that prediction market is unbiased under two extreme and separate conditions on the utility functions and the distribution of beliefs: either as in Proposition 2 when the utility is logarithmic $(P=2 A)$ or as in Proposition 3 when mean beliefs equal one half for symmetric belief distributions.

When traders are risk neutral, Ottaviani and Sorensen (2015, Proposition 2 and Corollary 1) show that underreaction leads to the favorite-longshot bias under the assumption of bounded wealth. They show that underreaction and hence the favorite-longshot bias becomes more pronounced for wider heterogeneous beliefs (measured by mean preserving spread). When traders are risk averse, Ottaviani and Sorensen (2015, Proposition 6) show a sufficient condition on price underreaction to information is the strict DARA, which is equivalent to $P>A$. They further illustrate the favorite-longshot bias for logarithmic utility. For CARA utility characterized by $P=A$, they show that there is no price underreaction. For all symmetric beliefs, the condition $P>2 A$ for the favorite-longshot bias in Proposition 4 is not only sufficient but also necessary. This result is different from Ottaviani and Sorensen (2015, Proposition 6). In particular, when $A \leq P<2 A$ (including CARA utility), we obtain a reverse favorite-longshot bias. Therefore our results on the favorite-longshot bias in Proposition 4 complements the price underreaction to information in Ottaviani and Sorensen (2015).

One may wonder whether the condition $(1 / 2-E \widetilde{p})(P(w)-2 A(w)) \geq 0$ is also necessary and sufficient for all distributions $\widetilde{p}$, not only symmetric ones. To see this, note first that $\pi^{*} \geq E \widetilde{p}$ is equivalent to $E \alpha(\widetilde{p}, \bar{p}) \geq 0$, and since $\alpha(\bar{p}, \bar{p})=0$, by Jensen's inequality the necessary and sufficient condition for all $\widetilde{p}$ is simply given by $\alpha_{p p}(p, \bar{p}) \geq 0$ for all $p$ 
and $\bar{p}$. The computation of $\alpha_{p p}(p, p)$ (in (A.1) of Appendix A) shows that the condition $(1 / 2-E \widetilde{p})(P(w)-2 A(w)) \geq 0$ is indeed necessary for the favorite-longshot bias. However this condition is not sufficient, as the following example shows.

Example 4 (Nonsymmetric beliefs): Consider two groups of traders with $u(w)=\sqrt{w}$ (i.e., $P>2 A$ ) and heterogeneous beliefs $p_{1}=0.1$ and $p_{2}=0.9$. When the proportion of traders with beliefs $p_{1}=0.1$ is $75 \%$ then $\pi^{*}=0.272<E \widetilde{p}=0.3$ (i.e., the longshot is undervalued), and when the proportion of traders with beliefs $p_{1}=0.1$ is $25 \%$ then $\pi^{*}=0.727>E \widetilde{p}=0.7$ (i.e., the favorite is overvalued).

We believe that the general results and selected numerical examples presented so far provide a fairly complete picture of the properties of equilibrium prices of a prediction market in the two-state case. This is the case that has been usually considered in the handful of theoretical papers on prediction markets that we have found in the literature. We next extend our discussion to a prediction market with more than two states.

\section{A discussion on the prediction market with many states}

We have so far considered a prediction market with only two states. In this section we provide a discussion on unbiased prediction market for any finite number $S$ of states with $S>2$. We examine whether the previous results obtained for two states can be generalized to the case with more than two states. Consistent with previous notations, we denote by the vector $\mathbf{p}_{i}=\left(p_{i 1}, \ldots, p_{i s}, \cdots, p_{i S}\right)$ the trader $i$ 's beliefs over states $s=1, \ldots, S$ for $i=1,2, \cdots, N$, and by $\pi_{s}$ the equilibrium price of state $s$. Similar to the two-state case, the prediction market is unbiased for state $s$ when $\pi_{s}=\frac{1}{N} \sum_{i=1}^{N} p_{i s}$.

As indicated in Section 3, a well-known result in the literature is that under a logarithmic utility function $u(w)=\log w$ prediction market is unbiased in the 2 -state case 
(Gjerstad 2004, Wolfers and Zitzewitz 2006). It turns out that this sufficiency result can be generalized in the sense that all $S$ state prices equal the mean of traders' beliefs for each state under $u(w)=\log w$. Since we have shown in Section 3 that $u(w)=\log w$ is also necessary for prediction market to be unbiased in the 2 -state case, we can therefore state the following result.

Proposition 5 (Generalization of Proposition 2) Prediction market is unbiased for all distributions of beliefs in the general $S$-state case if and only if $u(w)=\log w$.

This result should not suggest, however, that the previous results can be directly generalized to the $S$-state case. Consider the previous observation that if all traders have the same beliefs about a particular state (and hence the same beliefs about the other state), then the market is unbiased. This result is no longer valid in the general case. Indeed, we next show with the help of an example that, even if all traders have the homogeneous belief over one state, the equilibrium price of that state could nevertheless be different from its homogeneous belief.

Example 5 (Prediction market bias under homogeneous beliefs of a state): Let $N=2$ and $S=3$, and assume the following traders' beliefs: $\mathbf{p}_{1}=(1-2 p, p-\epsilon, p+\epsilon)$ and $\mathbf{p}_{2}=(1-2 p, p+\epsilon, p-\epsilon)$. Observe that the two traders have homogeneous beliefs $1-2 p$ over state 1 . Under CARA $u(w)=-e^{-r w}$ with $r>0$, we have however $\pi_{1}=\frac{1-2 p}{1-2 p+2 \sqrt{p^{2}-\epsilon^{2}}}>1-2 p$ for any $\epsilon \neq 0$.

We now make another observation using the previous example. With the same numerical values for individual beliefs and utilities, we consider an alternative prediction market. Assume that there are only two Arrow-Debreu assets: an asset that pays $\$ 1$ if state 1 occurs, and another asset that pays $\$ 1$ if either state 2 or state 3 occurs. We have therefore a binary prediction market. But since the traders' beliefs over the two states $(1-2 p, 2 p)$ are now homogeneous, we obviously retrieve the unbiased prediction market. Therefore, this simple observation shows that the equilibrium state price varies depending 
on the number of states on which it is possible to bet. In other words, this means that the "design" of the prediction market matters for equilibrium state prices. ${ }^{10}$ This makes sense since the design of the prediction market may affect trading opportunities under heterogeneous beliefs. Following this observation, one may ask: when is an equilibrium state price always independent from the design of the prediction market? It can be easily shown that this is the case when all traders believe that all other $S-1$ states are equally likely (see Proposition 6 below).

Example 5 also shows that the equilibrium price of one state depends on the distribution of beliefs in other states (through the parameter $\epsilon$ ). Note that this is inconsistent with a result in Varian (1985) stating that the equilibrium price in state $s$ depends only on the distributions of subjective probabilities that the state will occur. ${ }^{11}$ One may therefore ask: when does the equilibrium price of one state depend only on the beliefs about that state? Interestingly the answer to this question is exactly the same as the one to the question asked above about the prediction market design. Indeed, if the equilibrium price of one state in a $S$-state prediction market is always equal to the equilibrium price of that state in a prediction market, this precisely means that the distribution of beliefs in all the other states is irrelevant for that equilibrium state price. We state this result in the following proposition.

Proposition 6 For all $u$, the equilibrium price of one state, say $\pi_{1}$ for $s=1$, in a $S$-state prediction market only depends on the beliefs about that state $p_{i 1}$ if and only if

\footnotetext{
${ }^{10}$ To illustrate this interpretation, consider the horse race illustration. Example 5 and the last observation indicate that in a race with $S>2$ horses, the equilibrium state price that one specific horse wins the race depends on whether it is possible to place bets separately on each of the other horses participating in the race.

${ }^{11}$ Varian's result is obtained in a $S$-state complete market setting with heterogeneous beliefs and heterogeneous initial wealth. Our prediction market model in this section is thus a particular case of Varian's model since we assume a common initial wealth (thus removing any initial risk-sharing motivations for trade). Therefore Varian's result should also apply to our simpler setting. Note that Ingersoll (1987, p. 214) also presents a similar result as that of Varian: "The Arrow-Debreu price for an insurable state depends only on agregate wealth in that state and the pattern of beliefs about that state".
} 
$p_{i s}=p_{i}$ for all $s=2, \ldots, S$, and for all $i=1, \ldots, N$ (i.e. if and only if states 2 to $S$ are judged as equally likely by all traders).

Example 5 has shown that prediction market may be biased despite homogeneous beliefs in that state. The next example shows the dual result that prediction market may be unbiased despite heterogeneous beliefs. Specifically, Example 6 identifies a case with symmetric beliefs where prediction market is unbiased for any CRRA utility functions.

Example 6 (Prediction market is unbiased under heterogeneous beliefs): Let $N=2$ and $S=3$, and assume the following traders' beliefs: $\mathbf{p}_{1}=(1 / 2+1 / 4,1 / 3-1 / 6,1 / 6-1 / 12)$ and $\mathbf{p}_{2}=(1 / 2-1 / 4,1 / 3+1 / 6,1 / 6+1 / 12)$. Then under $u(w)=w^{1-\gamma} /(1-\gamma)$ with $\gamma>0$, prediction market is unbiased for all states, i.e., $\pi_{1}=1 / 2, \pi_{2}=1 / 3$ and $\pi_{3}=1 / 6$.

Proposition 4 implies for symmetric beliefs of two-state and under CRRA utility functions that there is underpricing if and only if $\gamma>1$, and thus that prediction market is unbiased only in the knife-edge logarithmic case, i.e. $\gamma=1$. In contrast, Example 6 illustrates that the equilibrium state price can be an unbiased estimation of the mean beliefs even when beliefs are symmetric and $P \neq 2 A$. Hence the prediction market bias in $S$-state markets can be very different from 2-state markets.

\section{Conclusion}

In the last decades, academics as well as private-sector operators have increasingly used financial prediction markets with the primary objective to better predict future uncertain events. But under which conditions should prediction markets be biased?

This paper has derived generic theoretical conditions so that equilibrium state prices in prediction markets is biased from the mean of the beliefs of traders. We have shown that even in a setting with homogenous traders, the conditions on risk preferences and/or on distributions of beliefs that lead to biased prediction market. We also provided a set of additional conditions that are informative about how prediction market prices vary with 
the belief distributions and the utility functions of participants as well as with the "design" of the prediction market. In particular, the paper has identified an exact condition on risk aversion such that the favorite-longshot bias (Ali 1977) always holds for any symmetric belief distributions, and an exact condition on beliefs such that the equilibrium price of one state only depends on the heterogeneity of beliefs about that state (Varian 1985).

A central assumption in our model is that traders do not update their subjective probabilities. With that assumption, we have followed the common setting adopted in the early economic papers on prediction markets (Gjerstad 2004, Manski 2006, Wolfers and Zitzewitz 2006). We believe that we have in turn provided in this paper a fairly complete picture of prediction market bias in this specific setting. In a set of important papers, Ottaviani and Sorensen $(2009,2010,2015)$ provide an alternative informational explanation to the prediction market bias without relaxing standard Bayesian updating. For sure, in many environments, investors do update their beliefs based on new information. On the other hand, there is a lot of evidence that people are not Bayesian. Typically, the extensive research on the "confirmation bias" in psychology show that people tend to search for, interpret or recall information in a way that confirms their prior beliefs. This suggests that the mechanism underlying the prediction market bias may depend on the specific prediction market characteristics and participants. Ultimately, this is an empirical question. Since our paper identifies a simple mechanism that relates the prediction market bias to traders' risk preferences, we believe that it can provide a good basis for empirical testing. 


\section{Appendix A: Proof of Propositions 1-4}

\section{A.1 Proof of Proposition 1}

We are done if we can show that the second term of the right hand side in (6) is negative. As this is simple to show, we only provide a sketch of the proof. Let $\widetilde{x}=(1-\pi,-\pi ; p)$ denote a random variable $\widetilde{x}$ which takes values of $1-\pi$ and $-\pi$ with probabilities $p$ and $1-p$, respectively. Then the first order condition (2) can be written more compactly $E\left[\widetilde{x} u^{\prime}(w+\alpha \widetilde{x})\right]=0$. We thus are done if we can show that this last equality implies $-\alpha E\left[\widetilde{x} u^{\prime \prime}(w+\alpha \widetilde{x})\right] \leq 0$, that is, the second term of the right hand side in (6) is negative. Then it is direct to see that this implication means that $-u^{\prime}$ is more risk averse than $u$, which is equivalent to nonincreasing absolute risk aversion.

\section{A.2 Proof of Proposition 2}

We just need to prove the necessity. Namely, let $\bar{p}=E \widetilde{p}$, we must show that if $E \alpha(\widetilde{p}, \bar{p})=0$ for all $\widetilde{p}$, then $u(w)=\log w$. Since $E \alpha(\widetilde{p}, \bar{p})=0$ for all belief distribution $\widetilde{p}$, this must also hold for a specific class of probability distribution in $\widetilde{p}$. We consider the class of "small" risk, that is we assume that $\widetilde{p}$ is close enough to $\bar{p}$ in the sense of a second-order approximation: $E \alpha(\widetilde{p}, \pi)=\alpha(\bar{p}, \pi)+0.5 E(\widetilde{p}-\bar{p})^{2} \alpha_{p p}(\bar{p}, \pi)$. Using this last equality, the necessary condition $E \alpha(\widetilde{p}, \bar{p})=0$ implies $\alpha(\bar{p}, \bar{p})+0.5 E(\widetilde{p}-\bar{p})^{2} \alpha_{p p}(\bar{p}, \bar{p})=0$. Since for all $p$, we have $\alpha(p, p)=0$, the necessary condition becomes $\alpha_{p p}(p, p)=0$. Differentiating again (3) with respect to $p$ to compute $\alpha_{p p}(p, p)$ we obtain

$$
\begin{aligned}
0 & =2 \alpha_{p}(p, \pi)\left\{(1-\pi)^{2} u^{\prime \prime}(w+\alpha(p, \pi)(1-\pi))-\pi^{2} u^{\prime \prime}(w-\alpha(p, \pi) \pi)\right\} \\
& +\alpha_{p p}(p, \pi)\left\{p(1-\pi)^{2} u^{\prime \prime}(w+\alpha(p, \pi)(1-\pi))+(1-p) \pi^{2} u^{\prime \prime}(w-\alpha(p, \pi) \pi)\right\} \\
& +\alpha_{p}(p, \pi)^{2}\left\{p(1-\pi)^{3} u^{\prime \prime \prime}(w+\alpha(p, \pi)(1-\pi))-(1-p) \pi^{3} u^{\prime \prime \prime}(w-\alpha(p, \pi) \pi)\right\} .
\end{aligned}
$$


Taking $\pi=p$ in the last expression, we have $\alpha_{p}(p, p)=\frac{1}{p(1-p)} \times \frac{u^{\prime}(w)}{-u^{\prime \prime}(w)}$ from $(4)$, then rearranging yields

$$
\alpha_{p p}(p, p)=\frac{(1-2 p)}{p^{2}(1-p)^{2}}\left[\frac{u^{\prime}(w)}{u^{\prime \prime}(w)}\right]^{2}\left[\frac{u^{\prime \prime \prime}(w)}{-u^{\prime \prime}(w)}-2 \frac{-u^{\prime \prime}(w)}{u^{\prime}(w)}\right] .
$$

Therefore a necessary condition is $\frac{u^{\prime \prime \prime}(w)}{-u^{\prime \prime}(w)}=2 \frac{-u^{\prime \prime}(w)}{u^{\prime}(w)}$. Finally, integrating this differential equation gives $u(w)=\log w$.

\section{A.3 Proof of Proposition 3}

We want to show that if $\widetilde{p}$ is symmetric about $1 / 2$ then $E \widetilde{p}=\pi^{*}$ for all $u$. Observe from the first order condition (2) that $\alpha(p, \pi)=-\alpha(1-p, 1-\pi)$. This implies that the equilibrium condition can be written $E \alpha\left(\widetilde{p}, \pi^{*}\right)=E \alpha\left(1-\widetilde{p}, 1-\pi^{*}\right)=0$. Observe then that $\widetilde{p}$ symmetric about $1 / 2$ means that $\widetilde{p}$ is distributed as $1-\widetilde{p}$. Consequently the equilibrium condition implies $E \alpha\left(\widetilde{p}, \pi^{*}\right)=E \alpha\left(\widetilde{p}, 1-\pi^{*}\right)$. Since the equilibrium is assumed to be unique, this last condition implies $\pi^{*}=1-\pi^{*}$, that is $\pi^{*}=1 / 2=E \widetilde{p}$.

\section{A.4 Proof of Proposition 4}

Recall that, when the equilibrium is unique, $\pi^{*} \geq \bar{p}$ if and only if $E \alpha(\widetilde{p}, \bar{p}) \geq 0$. For symmetric distributions, this holds true if and only if for all $\bar{p}$ (hereafter denoted $p$ ) we have

$$
g(\delta)=\alpha(p+\delta, p)+\alpha(p-\delta, p) \geq 0
$$

in which $\alpha(p+\delta, p)$ is the unique solution of

$$
(p+\delta)(1-p) u^{\prime}(w+\alpha(p+\delta, p)(1-p))-(1-p-\delta) p u^{\prime}(w-\alpha(p+\delta, p) p)=0
$$

and $\alpha(p-\delta, p)$ is the unique solution of

$$
(p-\delta)(1-p) u^{\prime}(w+\alpha(p-\delta, p)(1-p))-(1-p+\delta) p u^{\prime}(w-\alpha(p-\delta, p) p)=0
$$


for $\delta \in[0, \min \{p, 1-p\}]$.

Observe that $g(0)=0$ and $g^{\prime}(0)=0$. Moreover, we have $g^{\prime \prime}(0)=2 \alpha_{p p}(p, p)$. Then, taking $\alpha_{p p}(p, p)$ from (A.1), we can see that $g^{\prime \prime}(0) \geq 0$ is equivalent to $(1 / 2-p)(P(w)-$ $2 A(w)) \geq 0$ for all $w$. This provides the necessity part of the Proposition.

We now prove the sufficiency. From (A.4), condition (A.2) is equivalent to

$$
(p-\delta)(1-p) u^{\prime}(w-\alpha(p+\delta, p)(1-p))-(1-p+\delta) p u^{\prime}(w+\alpha(p+\delta, p) p) \geq 0 .
$$

Denoting $\phi(x)=1 / u^{\prime}(x)$ and $\alpha=\alpha(p+\delta, p) \geq 0, \pi^{*} \geq p$ is therefore satisfied if

$$
(p+\delta)(1-p) \phi(w-\alpha p)-(1-p-\delta) p \phi(w+\alpha(1-p))=0
$$

implies

$$
(p-\delta)(1-p) \phi(w+\alpha p)-(1-p+\delta) p \phi(w-\alpha(1-p)) \geq 0
$$

We now introduce two random variables:

$$
\tilde{x}=\left\{\begin{array}{ll}
w+\alpha p, & \frac{p-\delta}{2 p} \\
w-\alpha p, & \frac{p+\delta}{2 p}
\end{array} \quad, \quad \tilde{y}=\left\{\begin{array}{ll}
w+\alpha(1-p), & \frac{1-p-\delta}{2(1-p)} \\
w-\alpha(1-p), & \frac{1-p+\delta}{2(1-p)}
\end{array} .\right.\right.
$$

Then it can be verified that $E \tilde{x}=E \tilde{y}=w-\alpha \delta$ and $\tilde{x}$ is a mean-preserving spread of $\tilde{y}$ if and only if $p \geq 1 / 2$. Note that $\phi^{\prime \prime}(x) \geq 0$ if and only if $P \leq 2 A$. Therefore, when $p \geq 1 / 2$ and $P \leq 2 A$, we have

$$
E \phi(\tilde{x}) \geq E \phi(\tilde{y})
$$

which is equivalent to

$$
\begin{aligned}
& \frac{1}{2 p}[(p-\delta) \phi(w+\alpha p)+(p+\delta) \phi(w-\alpha p)] \\
\geq & \frac{1}{2(1-p)}[(1-p-\delta) \phi(w+\alpha(1-p))+(1-p+\delta) \phi(w-\alpha(1-p))] .
\end{aligned}
$$

This last inequality then leads to

$$
\begin{aligned}
& (1-p)(p-\delta) \phi(w+\alpha p)-p(1-p+\delta) \phi(w-\alpha(1-p)) \\
\geq & -[(1-p)(p+\delta) \phi(w-\alpha p)-p(1-p-\delta) \phi(w+\alpha(1-p))]=0,
\end{aligned}
$$


where the last equality is given by (A.6). This shows that the condition (A.7) is satisfied. Hence $\pi^{*} \geq p$ when $p \geq 1 / 2$ and $P \leq 2 A$. Moreover, when $p \leq 1 / 2, \tilde{y}$ is a mean-preserving spread of $\tilde{x}$, and $\phi^{\prime \prime}(x) \leq 0$ is equivalent to (A.8), leading to $\pi^{*} \geq p$. The case $\pi^{*} \leq p$ under $(1 / 2-p)(P-2 A) \leq 0$ can be demonstrated in an analogous fashion. This concludes the proof.

\section{Appendix B: Proof of Results in Section 5}

In this appendix, we setup a $S$-state prediction market, derive the equilibrium state prices, and provide the proofs of the propositions and details of the examples in Section 5.

\section{B.1 The $S$-state model}

Consider a prediction market with $N$ traders, indexed by $i=1, \cdots, N$, and $S$ states, indexed by $s=1, \cdots, S$. Traders have the same utility function $u(\cdot)$, however they have heterogeneous beliefs in the probability distribution over the states of nature, denoted by $\mathbf{p}_{i}=\left(p_{i 1}, p_{i 2}, \cdots, p_{i S}\right)$ for trader $i$. Let $\pi_{s}$ be the price of the Arrow-Debreu asset $s$ that delivers $\$ 1$ in state $s$ and $\$ 0$ in other states for $s=1, \cdots, S$. Trader $i$ chooses a portfolio $\alpha_{i}=\left(\alpha_{i 1}, \cdots, \alpha_{i S}\right)$ of the Arrow-Debreu assets to maximize his expected utility of portfolio wealth based on his belief $\mathbf{p}_{i}$. This leads to the standard first-order condition (FOC):

$$
p_{i s} u_{i}^{\prime}\left(w_{i s}\right)=\lambda_{i} \pi_{s}
$$

where $\lambda_{i}$ is the Lagrange multiplier,

$$
w_{i s}=w_{o}+\alpha_{i s}-\sum_{j=1}^{S} \pi_{j} \alpha_{i j}, \quad i=1, \cdots, N ; \quad s=1, \cdots, S
$$

is the portfolio wealth of trader $i$ in state $s$, and $w_{o}$ is the initial wealth. 


\section{B.2 The equilibrium state prices}

Based on the setup in subsection B.1, the equilibrium state prices $\left\{\pi_{s}\right\}$ are determined by the market clearing condition

$$
\sum_{i=1}^{N} \alpha_{i s}=0, \quad s=1, \cdots, S .
$$

To derive the equilibrium state prices, we consider three types of utility functions and the results are summarized in three Lemmas.

Lemma 1. For $u(x)=\log (x)$, the equilibrium state prices are given by

$$
\pi_{s}=\frac{1}{N} \sum_{i=1}^{N} p_{i s}, \quad s=1, \cdots, S
$$

that is, the state price is the mean probability belief of traders in the state.

Proof: With $u(x)=\log (x)$, the FOC (B.1) becomes

$$
w_{o}+\alpha_{i s}-\sum_{k=1}^{S} \pi_{k} \alpha_{i k}=\frac{1}{\lambda_{i}} \frac{p_{i s}}{\pi_{s}}, \quad i=1, \cdots, N ; s=1, \cdots, S,
$$

which leads to

$$
\alpha_{i s}=\alpha_{i S}+\frac{1}{\lambda_{i}}\left(\frac{p_{i s}}{\pi_{s}}-\frac{p_{i S}}{\pi_{S}}\right), \quad s=1, \cdots, S .
$$

Substituting the above expressions into (B.3) for $s=S$, we obtain that $\lambda_{i}=w_{o}$. This implies that the Lagrange multiplier $\lambda_{i}$ is a constant in this case. Applying the market clearing condition to (B.3) then leads to the equilibrium state prices (B.2).

Lemma 2. For CARA utility $u(x)=-e^{-r x} / r$ with $r>0$, the equilibrium state prices are given by

$$
\pi_{s}=\frac{p_{s}^{*}}{\sum_{k=1}^{S} p_{k}^{*}} \quad \text { with } p_{s}^{*}=\left(\prod_{i=1}^{N} p_{i s}\right)^{1 / N} \quad \text { for } s=1, \cdots, S
$$


Proof: With $u(x)=-e^{-r x} / r$, the FOC (B.1) becomes

$$
\frac{p_{i s}}{\pi_{s}} e^{-r w_{i s}}=\lambda_{i}, \quad i=1, \cdots, N, \quad s=1, \cdots, S .
$$

This leads to

$$
\alpha_{i s}-\sum_{k=1}^{S} \pi_{k} \alpha_{i k}=-w_{o}+\frac{1}{r}\left[\log \left(\frac{p_{i s}}{\pi_{s}}\right)-\log \left(\lambda_{i}\right)\right], \quad s=1, \cdots, S .
$$

Applying the market clearing conditions to (B.5), we obtain

$$
r w_{o}+\frac{1}{N} \sum_{i=1}^{N} \log \left(\lambda_{i}\right)=\log \left(\frac{p_{s}^{*}}{\pi_{s}}\right), \quad s=1, \cdots, S,
$$

where $p_{s}^{*}$ is defined by $\log \left(p_{s}^{*}\right)=\frac{1}{N} \sum_{i=1}^{N} \log \left(p_{i s}\right)$ for $s=1, \cdots, S$. Also, from (B.5),

$$
\alpha_{i s}=\alpha_{i S}+\frac{1}{r}\left[\log \left(\frac{p_{i s}}{\pi_{s}}\right)-\log \left(\frac{p_{i S}}{\pi_{S}}\right)\right] \text {, }
$$

for $s=1, \cdots, S$. Substituting (B.7) into (B.5) for $s=S$, we have

$$
\sum_{s=1}^{S} \pi_{s} \log \left(\frac{p_{i s}}{\pi_{s}}\right)=r w_{o}+\log \left(\lambda_{i}\right), \quad i=1, \cdots, N
$$

Aggregating (B.8) over $i$ then leads to

$$
r w_{o}+\frac{1}{N} \sum_{i=1}^{N} \log \left(\lambda_{i}\right)=\sum_{s=1}^{S} \pi_{s} \log \left(\frac{p_{s}^{*}}{\pi_{s}}\right) .
$$

Substituting (B.9) into (B.6), we then have

$$
\log \left(\frac{p_{s}^{*}}{\pi_{s}}\right)=\sum_{k=1}^{S} \pi_{k} \log \left(\frac{p_{k}^{*}}{\pi_{k}}\right), \quad s=1, \cdots, S .
$$

Therefore $p_{s}^{*} / \pi_{s}=\beta$ is a constant, independent of the state. Then (B.4) follows from $\sum_{s=1}^{S} p_{s}^{*}=\sum_{s=1}^{S} \pi_{s} \beta=\beta$.

Lemma 3. For CRRA utility $u(x)=x^{1-\gamma} /(1-\gamma)$ with $\gamma \neq 1$. the equilibrium state prices $\pi_{s}$ satisfy

$$
\pi_{s}^{1 / \gamma}=\frac{1}{N} \sum_{i=1}^{N} \frac{p_{i s}^{1 / \gamma}}{\sum_{k=1}^{S} \pi_{k}\left(\frac{p_{i k}}{\pi_{k}}\right)^{1 / \gamma}}, \quad s=1, \cdots, S
$$


Proof: With the CRRA utility function, the FOC (B.1) becomes $u^{\prime}\left(w_{i s}\right)=w_{i s}^{-\gamma}=$ $\lambda_{i} \pi_{s} / p_{i s}$. Hence, with $g=-1 / \gamma, w_{i s}=\left(\frac{\lambda_{i}}{p_{i s}} \pi_{s}\right)^{g}$. This, together with $w_{i s}=w_{o}+$ $\alpha_{i s}-\sum_{k=1}^{S} \pi_{k} \alpha_{i k}$, leads to

$$
\alpha_{i s}=-w_{o}+\sum_{k=1}^{S} \pi_{k} \alpha_{i k}+\left(\frac{\lambda_{i}}{p_{i s}} \pi_{s}\right)^{g} .
$$

Equation (B.11) implies that, for $s=1, \cdots, S$,

$$
\alpha_{i s}=\alpha_{i S}+\left[\left(\frac{\lambda_{i}}{p_{i s}} \pi_{s}\right)^{g}-\left(\frac{\lambda_{i}}{p_{i S}} \pi_{S}\right)^{g}\right] .
$$

Substituting (B.12) into (B.11) for $s=S$ and using the market clear condition, we obtain

$$
w_{o}=\sum_{s=1}^{S} \pi_{s}\left(\frac{\lambda_{i}}{p_{i s}} \pi_{s}\right)^{g}, \quad i=1, \cdots, N
$$

Also, applying the market clearing condition to (B.11), we have

$$
w_{o}=\frac{1}{N} \sum_{i=1}^{N}\left(\frac{\lambda_{i}}{p_{i s}} \pi_{s}\right)^{g}, \quad s=1, \cdots, S .
$$

Combining (B.13) with (B.14) leads to the state prices $\pi_{s}$ in (B.10).

\section{B.3 Proofs of Propositions 5 and 6}

The proof of Proposition 5 follows easily from the equilibrium state prices (B.2) in Lemma 1 for $u(x)=\log (x)$. To prove Proposition 6 , we first show that $p_{i s}=p_{i}$ for $s=2, \ldots, S$ and $i=1, \ldots, N$ implies $\pi_{2, S}=\sum_{s=2}^{S} \pi_{s}$, where $\pi_{2, S}$ denote the state price of an Arrow-Debreu security that delivers $\$ 1$ if either state $j=2, \ldots, S$ occurs and $\$ 0$ if state 1 occurs. If $p_{i s}=p_{i}$ for $s=2, \ldots, S$, we have from the FOC (B.1) that

$$
\frac{u^{\prime}\left(w_{i j}\right)}{u^{\prime}\left(w_{i k}\right)}=\frac{\pi_{j}}{\pi_{k}}, \text { for all } i=1, \ldots, N \text { and } j, k=2, \ldots, S .
$$

Assume then by contradiction that $\pi_{j}>\pi_{k}$ for some $j, k=2, \ldots, S$ and $j \neq k$. This implies $w_{i j}<w_{i k}$, and hence $\alpha_{i j}<\alpha_{i k}$ for all $i=1, \ldots, N$. The last inequality cannot hold at the equilibrium due to the market clearing condition. As a result we must have $\pi_{j}=\pi_{k} \equiv \pi$ for all $j=2, \ldots, S$. It is immediate that each trader $i$ must demand the same 
amount, say $\alpha_{i}$, for $j, k=2, \ldots, S$. The problem of each trader $i$ is then to select $\alpha_{i 1}$ and $\alpha_{i}$ to maximize

$$
p_{i 1} u\left(w+\alpha_{i 1}-\alpha_{i 1} \pi_{1}-\alpha_{i}(S-1) \pi\right)+\left(1-p_{i 1}\right) u\left(w+\alpha_{i}-\alpha_{i 1} \pi_{1}-\alpha_{i}(S-1) \pi\right) .
$$

This is equivalent to a binary-prediction market in which $(S-1) \pi$ denotes the equilibrium price of an Arrow-Debreu security that delivers $\$ 1$ if either state $j=2, \ldots, S$ occurs. Therefore we have $\sum_{j=2}^{S} \pi_{j}=(S-1) \pi=\pi_{2, S}$. This implies the $S$-state problem is equivalent to a reduced two-state problem by combining states 2 to $S$ into one state. Thus the equilibrium price of state 1 only depends on the beliefs of the state.

We now show that if there exists an individual $i$ who assigns different probabilities for two states $j, k=2, \ldots, S$ then we may always have $\pi_{2, S} \neq \sum_{j=2}^{S} \pi_{j}$. Consider a simple example with 3 states and 2 traders, with the following structure of beliefs: $\mathbf{p}_{1}=$ $\left(1-2 p_{1}, p_{1}, p_{1}\right)$ and $\mathbf{p}_{2}=\left(1-2 p_{2}, p_{2}+e, p_{2}-e\right)$. Namely trader 1 judges states 2 and 3 as equally likely, while trader 2 judges states 2 and 3 as equally likely if and only if $e=0$. With CARA preferences, in the three-state prediction market we use the equilibrium state prices in (B.4) of Lemma 2 and obtain $\pi_{i}=p_{i}^{*} /\left(p_{1}^{*}+p_{2}^{*}+p_{3}^{*}\right)$ for $i=1,2,3$ with $p_{1}^{*}=\sqrt{\left(1-2 p_{1}\right)\left(1-2 p_{2}\right)}, p_{2}^{*}=\sqrt{p_{1}\left(p_{2}+e\right)}$ and $p_{3}^{*}=\sqrt{p_{1}\left(p_{2}-e\right)}$. However, in the two-state prediction market where states 2 and 3 are combined into one state, the beliefs $\mathbf{p}_{1}$ and $\mathbf{p}_{2}$ become $\overline{\mathbf{p}}_{1}=\left(1-2 p_{1}, 2 p_{1}\right)$ and $\overline{\mathbf{p}}_{2}=\left(1-2 p_{2}, 2 p_{2}\right)$, respectively. Hence the corresponding state prices become (using obvious notations) $\bar{\pi}_{1}=\bar{p}_{i}^{*} /\left(\bar{p}_{1}^{*}+\bar{p}_{2,3}^{*}\right.$ ) and $\bar{\pi}_{2,3}=\bar{p}_{2,3}^{*} /\left(\bar{p}_{1}^{*}+\bar{p}_{2,3}^{*}\right)$ with $\bar{p}_{1}^{*}=\sqrt{\left(1-2 p_{1}\right)\left(1-2 p_{2}\right)}$ and $\bar{p}_{2,3}^{*}=2 \sqrt{p_{1} p_{2}}$. Therefore $\bar{\pi}_{2,3}=\pi_{2}+\pi_{3}$ for all $p_{1}$ and $p_{2}$ if and only if $e=0$. A similar example can be generated for any arbitrary number of states. This completes the proof of Proposition 6.

\section{B.4 Proofs of the results in Examples 5 and 6}

In Example 5 with CARA utility function, we apply the equilibrium state price (B.4) in Lemma 2 to traders' beliefs and obtain that $p_{1}^{*}=1-2 p$ and $p_{2}^{*}=p_{3}^{*}=\sqrt{p^{2}-\epsilon^{2}}$, leading to the state price $\pi_{1}$ in the example. 
To show the result in Example 6, we apply the equilibrium state prices (B.10) in Lemma 3 for CRRA utility function. With $g=-1 / \gamma$, the state prices $\pi_{s}$ for $s=1,2,3$ in this case satisfy

$$
\begin{aligned}
& 2=\left(\pi_{1}\right)^{g}\left[p_{11}^{-g} / \Delta_{1}+p_{21}^{-g} / \Delta_{2}\right] \\
& 2=\left(\pi_{2}\right)^{g}\left[p_{12}^{-g} / \Delta_{1}+p_{22}^{-g} / \Delta_{2}\right] \\
& 2=\left(\pi_{3}\right)^{g}\left[p_{13}^{-g} / \Delta_{1}+p_{23}^{-g} / \Delta_{2}\right],
\end{aligned}
$$

where

$$
\begin{aligned}
& \Delta_{1}=\pi_{1}\left(\pi_{1} / p_{11}\right)^{g}+\pi_{2}\left(\pi_{2} / p_{12}\right)^{g}+\pi_{3}\left(\pi_{3} / p_{13}\right)^{g}, \\
& \Delta_{2}=\pi_{1}\left(\pi_{1} / p_{21}\right)^{g}+\pi_{2}\left(\pi_{2} / p_{22}\right)^{g}+\pi_{3}\left(\pi_{3} / p_{23}\right)^{g} .
\end{aligned}
$$

With the specified heterogeneous probabilities, $\Delta_{1}=(12)^{g} \delta_{1}$ and $\Delta_{2}=(12)^{g} \delta_{2}$, where

$$
\begin{aligned}
& \delta_{1}=\pi_{1}\left(\pi_{1} / 9\right)^{g}+\pi_{2}\left(\pi_{2} / 2\right)^{g}+\pi_{3}\left(\pi_{3}\right)^{g}, \\
& \delta_{2}=\pi_{1}\left(\pi_{1} / 3\right)^{g}+\pi_{2}\left(\pi_{2} / 6\right)^{g}+\pi_{3}\left(\pi_{3} / 3\right)^{g} .
\end{aligned}
$$

Correspondingly, equations (B.15)-(B.17) lead to

$$
\begin{aligned}
& 2\left(\pi_{1}\right)^{-g}=9^{-g} / \delta_{1}+3^{-g} / \delta_{2} \\
& 2\left(\pi_{2}\right)^{-g}=2^{-g} / \delta_{1}+6^{-g} / \delta_{2} \\
& 2\left(\pi_{3}\right)^{-g}=1 / \delta_{1}+3^{-g} / \delta_{2}
\end{aligned}
$$

From (B.19) and (B.20), we obtain $\pi_{3}=\pi_{2} / 2$. Hence

$$
\begin{aligned}
& \delta_{1}=\pi_{1}\left(\pi_{1} / 9\right)^{g}+(3 / 2) \pi_{2}\left(\pi_{2} / 2\right)^{g}, \\
& \delta_{2}=\pi_{1}\left(\pi_{1} / 3\right)^{g}+(3 / 2) \pi_{2}\left(\pi_{2} / 6\right)^{g} .
\end{aligned}
$$

Also, from (B.18) and (B.19),

$$
3^{g}\left[\delta_{1}+3^{g} \delta_{2}\right] \pi_{2}^{g}=2^{g}\left[3^{g} \delta_{1}+\delta_{2}\right] \pi_{1}^{g} .
$$

Substituting (B.21) and (B.22) into (B.23), we obtain

$$
\left[3^{g}+3^{-g}\right]\left[\pi_{1}-(3 / 2) \pi_{2}\right] \pi_{1}^{g} \pi_{2}^{g}=3(2 / 3)^{1+g} \pi_{2}^{1+2 g}\left[\left(\pi_{1} / \pi_{2}\right)^{1+2 g}-(3 / 2)^{1+2 g}\right],
$$

leading to $\pi_{1}=(3 / 2) \pi_{2}$. 
Acknowledgement-We thank Olivier Armantier, Kristoffer Glover, Christian Gollier, Bruce Grundy, Charles Manski, François Salanié, Lei Shi, Danny Yeung and Min Zheng, as well as seminar and conference participants at ESEM 2011, SCE 2011, BFS 2012, FIRN 2012, Paris-Dauphine and University of Technology Sydney (UTS) for comments. The first version of this paper was written while Nicolas Treich visited UTS in 2010. Financial support from the Quantitative Finance Research Center and the Paul Woolley Center at UTS is gratefully acknowledged. Tony He acknowledges funding for his subsequent visit in 2011 to Toulouse from the European Research Council under the European Community's Seventh Framework Programme (FP7/2007-2013) Grant Agreement no. 230589 and financial support from the Australian Research Council (ARC) under a Discovery Grant (DP130103210). Nicolas Treich also thanks the chair "Marché des risques et création de valeurs" Fondation du risque/SCOR. 


\section{References}

[1] Abel, A., 1989, Asset prices under heterogeneous beliefs: Implications for the equity premium. Mimeo, University of Pennsylvania.

[2] Ali, M., 1977, Probability and utility estimators for racetrack betters, Journal of Political Economy 85, 803-15.

[3] Armantier, O. and N Treich, 2013, Eliciting beliefs: Proper scoring rules, incentives, stakes and hedging, European Economic Review 62, 17-40.

[4] Arrow, K.J., Forsythe R.,Gorham M., Hahn R., Hanson R., Ledyard J.O., Levmore S., Litan R., Milgrom P., Nelson F.D., Neumann G.R., Ottaviani M., Schelling T.C.,Shiller R.J., Smith V.L.,Snowberg E., Sunstein C.R., Tetlock P.C., Tetlock P.E., Varian H.R., Wolfers J. and E. Zitzewitz, 2008, The promise of prediction markets, Science 320, 877-878.

[5] Berg, J., G. Neumann and T. Rietz, 2009, Searching for Google's value: Using prediction markets to forecast market capitalization prior to an initial public offering, Management Science 55, 348-361.

[6] Fishburn, P.C and R.B. Porter, 1976, Optimal portfolios with one safe and one risky asset: Effects of changes in rate of return and risk, Management Science 10, 10641073.

[7] Forsythe R., Nelson F., Neumann G.R. and J. Wright, 1992, Anatomy of an experimental political stock market, American Economic Review 82, 1142-61.

[8] Fountain, J. and G.W. Harrison, 2011, What do prediction markets predict?, Applied Economics Letters 18, 267-72.

[9] Gjerstad, S., 2004, Risk aversion, beliefs and prediction market equilibrium. Mimeo, University of Arizona Working Paper 04-17. 
[10] Goel, S., Reeves D. and D. Pennock, 2009, Collective revelation: A mechanism for self-verified, weighted, and truthful predictions, in Proceedings of the 10th ACM Conference on Electronic Commerce, 265-274.

[11] Gollier, C., 2001, The Economics of Risk and Time, MIT Press.

[12] Hahn R.W. and P.C. Tetlock, 2006, Introduction to Information Markets, in Information Markets: A New Way of Making Decisions, R.W. Hahn and P.C. Tetlock (eds), AEI-Brookings Joint Center for Regulatory Studies, Washington D.C..

[13] Healy P.J., Linardi S., Lowery R. and J. Ledyard, 2010, Prediction markets: Alternative mechanisms for complex environments with few traders, Management Science 56(11), 1977-1996.

[14] Hanson, R., 2006, Foul play in information markets, in Information Markets: A New Way of Making Decisions, R.W. Hahn and P.C. Tetlock (eds), AEI-Brookings Joint Center for Regulatory Studies, Washington D.C..

[15] Hong H. and J. Stein, 2007, Disagreement and the stock market, Journal of Economic Perspectives 21, 109-128.

[16] Ingersoll, J., 1987, Theory of Financial Decision-Making, Rowman and Littlefield, New Jersey.

[17] Jian, L. and R. Sami, 2012, Aggregation and manipulation in prediction markets: Effects of trading mechanism and information distribution, Management Science 58, 123-140.

[18] Jouini, E. and C. Napp, 2006, Aggregation of heterogeneous beliefs, Journal of Mathematical Economics 42, 752-770.

[19] Jouini, E. and C. Napp, 2007, Consensus consumer and intertemporal asset pricing with heterogeneous beliefs, Review of Economic Studies 74, 1149-1174. 
[20] Kimball, M.S., 1990, Precautionary savings in the small and in the large, Econometrica $61,53-73$.

[21] Manski, C.F., 2006, Interpreting the predictions of prediction markets, Economics Letters 91, 425-29.

[22] Manski, C. F., 2004, Measuring expectations, Econometrica 72, 1329-1376.

[23] Milgrom, P. and N. Stokey, 1982, Information, trade and common knowledge, Journal of Economic Theory 26(1), 17-27.

[24] Ottaviani, M. and P.N. Sorensen, 2009, Surprised by the parimutuel odds? American Economic Review, 99(5), 2129-34.

[25] Ottaviani, M. and P.N. Sorensen, 2010, Noise, information, and the favorite-longshot bias in parimutuel predictions, American Economic Journal: Microeconomics, 2(1), $58-85$.

[26] Ottaviani, M. and P.N. Sorensen, 2015, Price reaction to information with heterogeneous beliefs and wealth effects: underreaction, momentum, and reversal, American Economic Review, 105(1), 1-34.

[27] Page L. and R.T. Clemen, 2013, Do prediction markets produce well calibrated probability forecasts?, Economic Journal, 123, 491-513.

[28] Roche, H., 2011, Speculative bubbles in a pure exchange economy. Mimeo, California Polytechnic State University.

[29] Scheinkman, R.A. and W. Xiong, 2004, Heterogeneous beliefs, speculation and trading in financial markets, Lecture Notes in Mathematics 1847, Springer-Verlag, Berlin.

[30] Slovic, P., 2000, The Perception of Risk, Earthscan Publications.

[31] Surowiecki, J., 2005, The Wisdom of Crowds, Anchor Books. 
[32] Thaler, R.H. and W.T. Ziemba, 1988, Anomalies: Parimutual betting markets: racetrack and lotteries, Journal of Economic Perspectives 2, 161-74.

[33] Varian, H.R., 1985, Divergence of opinion in complete markets: A note, Journal of Finance XL, 309-317.

[34] Varian, H.R., 1989, Differences of opinion in financial markets, in Financial Risk: Theory, Evidence and Implications, Courtenay Stone (ed.), Kluwer Academic Publishers, Boston.

[35] Wolfers J. and E. Zitzewitz, 2004, Prediction markets, Journal of Economic Prespectives 18(2), 107-126.

[36] Wolfers J. and E. Zitzewitz, 2006, Interpreting prediction market price as probabilities, NBER working paper 12200. 